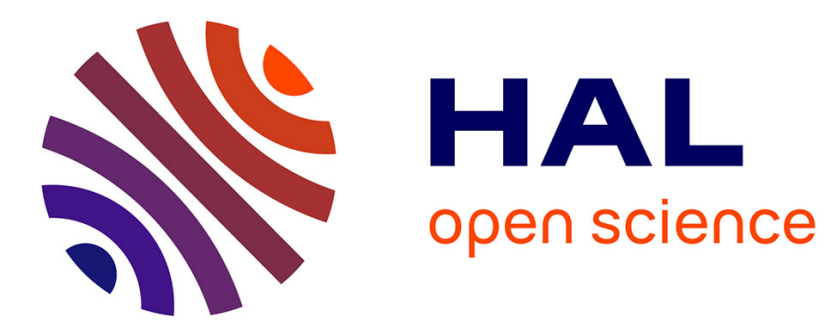

\title{
Enhanced thermal conductivity for mesophase pitch-based carbon fiber/modified boron nitride/epoxy composites
}

Benhui Fan, Yu Liu, Delong He, Jinbo Bai

\section{- To cite this version:}

Benhui Fan, Yu Liu, Delong He, Jinbo Bai. Enhanced thermal conductivity for mesophase pitchbased carbon fiber/modified boron nitride/epoxy composites. Polymer, 2017, 122, pp.71 - 76. 10.1016/j.polymer.2017.06.060 . hal-01842481

\section{HAL Id: hal-01842481 \\ https://hal.science/hal-01842481}

Submitted on 3 Dec 2020

HAL is a multi-disciplinary open access archive for the deposit and dissemination of scientific research documents, whether they are published or not. The documents may come from teaching and research institutions in France or abroad, or from public or private research centers.
L'archive ouverte pluridisciplinaire HAL, est destinée au dépôt et à la diffusion de documents scientifiques de niveau recherche, publiés ou non, émanant des établissements d'enseignement et de recherche français ou étrangers, des laboratoires publics ou privés. 


\title{
Enhanced Thermal Conductivity for Mesophase Pitch-Based Carbon Fiber /Modified Boron Nitride/Epoxy Composites
}

Benhui FAN, Yu LIU, Delong HE* and Jinbo BAI*

Laboratoire Mécanique des Sols, Structures et Matériaux (MSSMat), CNRS UMR

8579, Ecole CentraleSupelec, Université Paris Saclay, Grande Voie des Vignes, 92290, Chatenay-Malabry, France

Corresponding authors: Jinbo BAI, jinbo.bai@ecp.fr

Delong HE, delong.he@ecp.fr

\begin{abstract}
Carbon fiber reinforced polymer composites are important in advanced components which require high thermal conductivity (TC). However, the epoxy-rich interlayer makes even highly thermal conductive mesophase pitch-based carbon fibers (MPCF) less effective in the out-of-plane direction. In this study, boron nitride (BN) particles with high TC are incorporated in MPCF/epoxy composites and after an efficient surface treatment by coupling agent KH550, TC of composite has achieved 7.9 $\mathrm{W} /(\mathrm{m} * \mathrm{~K})$ which enhances $61 \%$ compared with the control sample without BN (4.9 $\left.\mathrm{W} /\left(\mathrm{m}^{*} \mathrm{~K}\right)\right)$. It is possibly attributed to the reinforced adhesion among modified BN $(\mathrm{mBN})$, epoxy and MPCF achieved by the silanol groups of KH550 which enables to reduce the thermal resistance as well as increase efficient packing between fillers and polymer matrix. Besides, TC of composites with $\mathrm{mBN}$ also is more stable with temperature's changing compared with the one incorporated by pristine $\mathrm{BN}$.
\end{abstract}

Key words: surface treatment, boron nitride, thermal conductivity, carbon fibers 


\section{Introduction}

Carbon fibers (CF) are the most promising materials in the field of aerospace and aeronautics due to the low density and high performances including the good mechanical property, flexible processability, and corrosion resistance, etc, compared with conventional materials like metal alloys or ceramics. ${ }^{[1,2]}$ Moreover, as an excellent thermal conductor, $\mathrm{CF}$ can also meet the requirements of high thermal conductivity (TC) for aerospace and aeronautics devices to guarantee efficient heat diffusion conduction and avoid locally substantial overheating and degradation of aircrafts. ${ }^{[3]}$ CF's thermal properties largely depend on the process condition and the organic precursors, namely the polyacrylonitrile (PAN)-based and the mesophase pitch (MP)-based. ${ }^{[4-6]}$ The mesophase pitch-based carbon fibers have a much higher in-plane TC (up to $1000 \mathrm{w} /\left(\mathrm{m}^{*} \mathrm{~K}\right)$ as reported) than that of PAN-based ones owning to the highly crystalline and oriented microstructure. ${ }^{[7,8]}$ Thus, the mesophase pitch-based carbon fibers (MPCF) are attractive for reinforcement materials in a variety of structural and functional components where the thermal conduction is highly required, such as artificial satellite antenna, heat sink and other thermal controls in electronics devices, etc. Unfortunately, the high TC of MPCF is largely limited once fabricated into composites due to the intrinsic low TC (around 0.2 $\mathrm{w} /(\mathrm{m} * \mathrm{~K}))$ and insolate nature of epoxy matrix. Therefore, it is highly desired to find an efficient way to improve the out-of-plane TC for the MPCF reinforced epoxy composites.

Enormous efforts and attempts have been endowed on how to address the problem of heat transfer in epoxy-rich zones of the composites. For instance, the liquid crystalline epoxy with high TC $1.16 \mathrm{w} /\left(\mathrm{m}^{*} \mathrm{~K}\right)$ is developed to improve TC of epoxy from the view of polymer's structure directly, ${ }^{[9,10]}$ but it is worthwhile to point out that the liquid crystalline epoxy usually has a high melting point which restricts the further application as a matrix for the composites. Another common and popular way is to prepare composites by incorporating fillers with high TC into the epoxy matrix including carbon based materials such as carbon nanotubes $(\mathrm{CNT}),{ }^{[11,12]}$ graphite nanoplatelets $(\mathrm{GNP}),{ }^{[13,14]}$ ceramic fillers such as boron nitride $(\mathrm{BN}),{ }^{[15,16]}$ 
aluminum nitride $(\mathrm{AlN})^{[17,18]}$ and metal particles ${ }^{[19,20]}$ such as silver, copper, etc. In order to achieve high TC for the composites, a large volume fraction of the fillers (over $50 \%)^{[1,15-18,24,25]}$ is usually needed but the enhancement on TC sometimes is slight since the interface adhesion between fillers and matrix is hard to be controlled well. The poor adhesion introduces more defects, voids and much area of interfaces which unavoidably lead to low TC for composites because the possibility of phonons scattering increases and then the heat transfer in the inner of composite is weakened. For the epoxy matrix, the heat transfer is mainly conducted by the energy quanta of atomic lattice vibration or phonon which is different from the metal by electrons as the charge carries. ${ }^{[1,2]}$ Hence, from the perspective of heat transfer, for pursuing a higher TC of MPCF/epoxy matrix composite, a better adhesion for fillers and epoxy matrix is the doubtlessly key factor for the composite to achieve high thermal property.

Surface treatment of fillers is a widely-used method to optimize the adhesion of fillers-matrix and modify fillers' dispersal. ${ }^{[21-23]}$ There are various surface modifiers for ameliorating filler's surface property and reducing thermal interfacial resistance with matrix including surfactants, coupling agents, functional polymers and inorganic coating. ${ }^{[1]}$ Experimental results have shown that an appropriate surface treatment for fillers including type's choosing and content's controlling enables to strengthen the interface regions' bonding. Furthermore, the surface modifiers can reduce the viscosity of the epoxy during the processing which can modify filler's dispersal in the epoxy. Even humble surface treatments for fillers may enhance the global TC for the composites as reported by researchers. ${ }^{[1]}$

In this study, in order to achieve high out-of-plane TC for the composite, MPCF, $\mathrm{BN}$ and epoxy are chosen as the raw materials to prepare composites (MPCF/BN/epoxy) with double layers structure. We firstly investigate different BN's volume fractions from $5 \%$ to $20 \%$ and moreover, an organo-silanes coupling agent (KH550) is applied for a surface treatment on BN particles (remarked as $\mathrm{mBN}$ ) so as to compare with $\mathrm{TC}$ of the composite incorporated by pristine $\mathrm{BN}$. The $\mathrm{TC}$ results illustrate that due to forming better interfacial adhesion after the surface treatment, the 
out-of-plane TC of composite with merely $20 \%$ volume fraction of $\mathrm{mBN}$ (MPCF/mBN-20\%/epoxy) has reached $7.9 \mathrm{w} /(\mathrm{m} * \mathrm{~K})$ which is much higher than that of control sample MPCF/epoxy $(4.9 \mathrm{w} /(\mathrm{m} * \mathrm{~K}))$. Besides, the TC of $\mathrm{MPCF} / \mathrm{mBN}-20 \% / e p o x y$ also shows good stability with the temperature changing.

\section{Experimental}

Materials: The polymer matrix used in this study is epoxy: the resin is $1080 \mathrm{~S}$ (Resoltech Ltd., France), bisphenol F-(epichlorhydrin) (number average molecular weight $<=700$ ). The curing agency is 1084 (Resoltech Ltd., France), 4-4'-methylenebis (cyciohexylamine) (MCHA). The mass ratio of resin and curing agent is $3: 1$. Boron nitride (BN) particles with the size of $1 \mu \mathrm{m}$ and the coupling agent, (3-Aminopropyl) triethoxysilane (KH550), are purchased from Sigma Aldrich directly. CW-300 is as the MPCF tissues used in the composite's preparation. MPCF tissue used in the study is ThermalGraph EWC-300X bought from Cytec and its TC are about $250-300 \mathrm{~W} /(\mathrm{mK})$.

Preparation of composites: The surface treatment for BN by KH550 can be found in elsewhere ${ }^{[24,25]}$ and in this study the addition of KH550 was 3\% calculating to BN's mass and the solvent used was ethanol. The preparation of the composite were divided into three steps: first, the mixture of fillers (BN or mBN) and resin with calculated weight fractions were mixed by three rolls mill for $20 \mathrm{~min}$ at the room temperature and after that the curing agent was dropped into the mixture. The obtained slurry of fillers, resin and curing agent was applied on two MPCF tissues carefully to form the composite with double layers. Second, the composite was put into two aluminum molds under vacuum for $30 \mathrm{~min}$ to remove the bubbles. Afterward, it was put into a vacuum bag and moved into a hot-press machine under the pressure of $0.8 \mathrm{~Pa}$ at $60{ }^{\circ} \mathrm{C}$ for $2 \mathrm{~h}$ with pump. Finally, the further curing of epoxy was conducted in an oven at $60{ }^{\circ} \mathrm{C}$ for $10 \mathrm{~h}$. The whole preparing process and epoxy's chemical reaction are presented in Fig.1. 


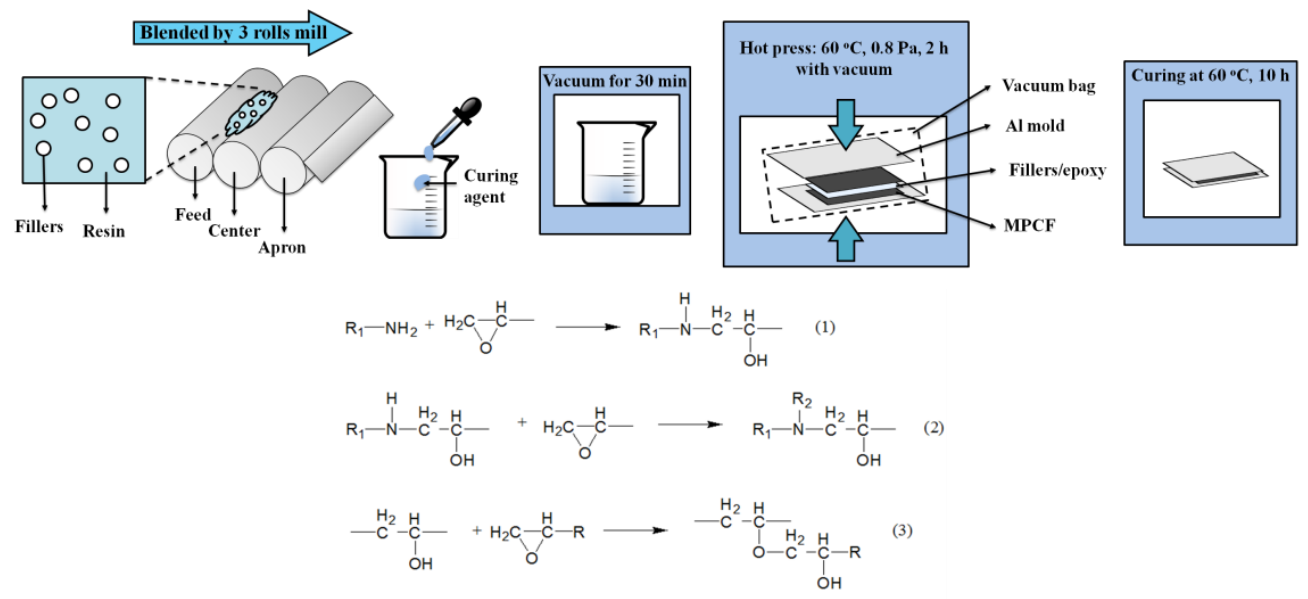

Figure 1. Schematic figure of the composite preparation process and the chemical reaction for epoxy matrix.

Characterization: The morphology for the surface fracture of the composite was conducted by scanning electron microscope (SEM) (LEO Gemini 530). Thermo gravimetric analysis (TGA) characterization was conducted by NETZSCH STA 449F3 in a wide temperature range from 30 to $800{ }^{\circ} \mathrm{C}$ with $10{ }^{\circ} \mathrm{C} / \mathrm{min}$ heating rate. The out-of-plane TC was calculated by flash method (Nanoflash LAF 440) at room temperature $\left(20{ }^{\circ} \mathrm{C}\right)$ and the evaluation was conducted by Cowan fitting with finite pulse correction. Dynamic mechanical analysis (DMA) for samples of the composite was conducted in three-point bending mode by NETZSCH DMA 242C. The measurement was from $30{ }^{\circ} \mathrm{C}$ to $150{ }^{\circ} \mathrm{C}$ and with $2{ }^{\circ} \mathrm{C} /$ min heating rate at $1 \mathrm{~Hz}$. The size of the sample for the measurement was $40 \mathrm{~mm} \times 10 \mathrm{~mm} \times 1 \mathrm{~mm}$. The loss tangent for the modulus is defined as $\tan \theta=\frac{E^{\prime \prime}}{E^{\prime}}$, where E' and E" correspond to the real and imaginary parts of the storage modulus E, respectively.

\section{Results and discussions}

The TGA for pristine $\mathrm{BN}$ and $\mathrm{mBN}$ are presented in Fig.2 (a). $\mathrm{mBN}$ in the blue line has a clear mass loss in the temperature range from 200 to $350{ }^{\circ} \mathrm{C}$ due to the addition of $\mathrm{KH} 550$ whose boiling point is about $217{ }^{\circ} \mathrm{C}$ while the pristine $\mathrm{BN}$ does not this in such a temperature range. This difference of the mass loss shows that KH550 is existed in $\mathrm{mBN}$ particles after simple surface treatment. We also do TGA for 
MPCF/BN-20\%/epoxy and MPCF/mBN-20\%/epoxy composites and it can be found the weight ratio between epoxy composite and MPCF is about 1:3 since the weight loss until $500{ }^{\circ} \mathrm{C}$ for both two composite is around $25 \%$.

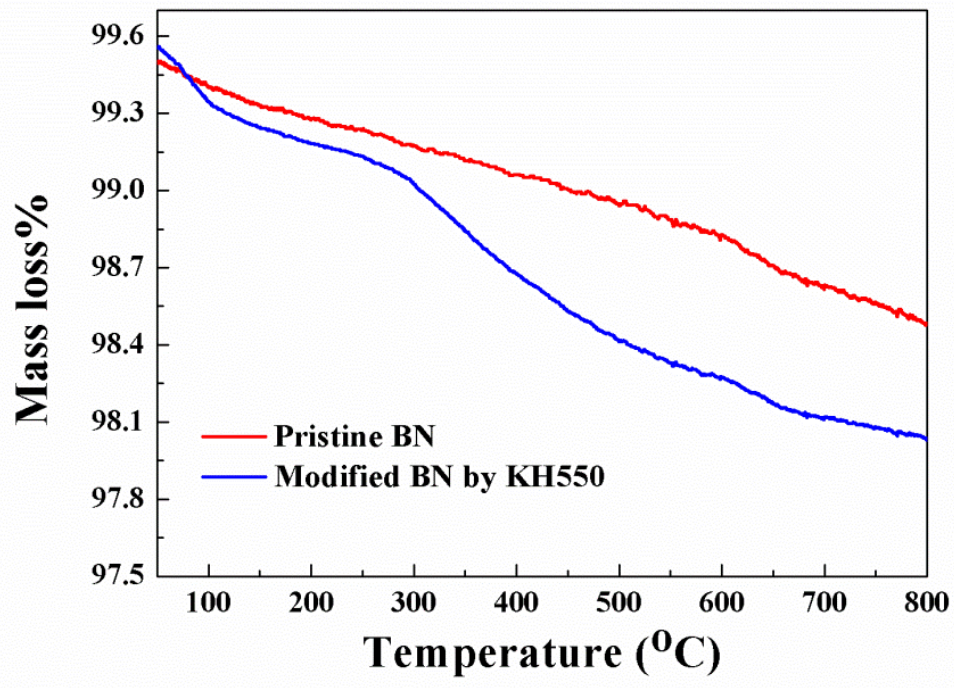

(a)

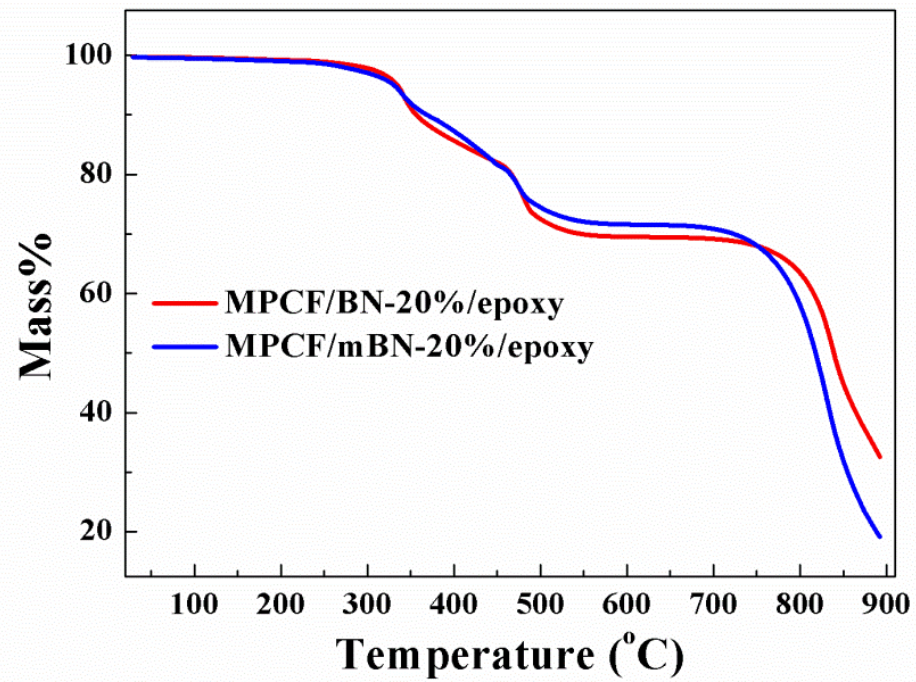

(b)

Figure 2. (a) TGA for pristine $\mathrm{BN}$ and $\mathrm{mBN}$ from 30 to $800{ }^{\circ} \mathrm{C}$. (b) TGA for two composites, MPCF/BN-20\%/epoxy and MPCF/mBN-20\%/epoxy from 30 to $900{ }^{\circ} \mathrm{C}$.

The SEM images of surface fractures for two kinds of composites with $20 \%$ volume fraction of filler's incorporation are presented in Fig.3. It can be found that 
MPCF can be adhered by epoxy with the fillers but the qualities of adhesion are different between MPCF/BN-20\%/epoxy and MPCF/mBN-20\%/epoxy. Compared with the Fig.3 (a) and Fig.3 (c), the adhesion in the boundary region shown in Fig.3 (c) of $\mathrm{MPCF} / \mathrm{mBN}-20 \% / \mathrm{epoxy}$ are better than that of MPCF/BN-20\%/epoxysince a denser structure can be figured out in Fig.3 (c). Meanwhile, observing Fig.3 (b) and Fig.3 (d), there are many little points remained in the surface of mBN in Fig.3 (d). Instead, the surface of $\mathrm{BN}$ is flat and clean in MPCF/BN-20\%/epoxy as shown in Fig.3 (b). These little points remained after epoxy's breaking indicate that $\mathrm{mBN}$ has formed modified bonding with epoxy matrix after treated by the KH550 coupling agent. But the clean and flat surface of $\mathrm{BN}$ infers a weak bonding with epoxy. Therefore, it can be viewed that MPCF/mBN-20\%/epoxy has better interfacial adhesion among MPCF, mBN and epoxy than that of MPCF/BN-20\%/epoxy.

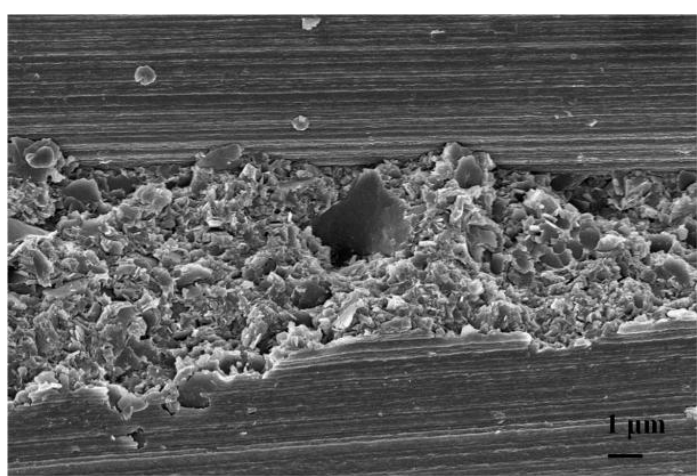

(a)

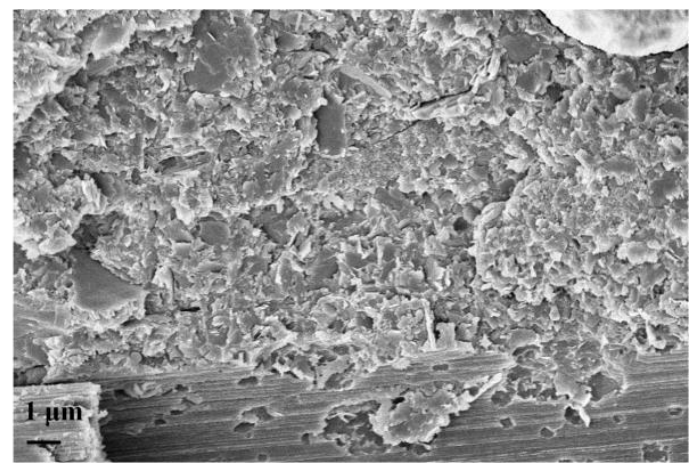

(c)

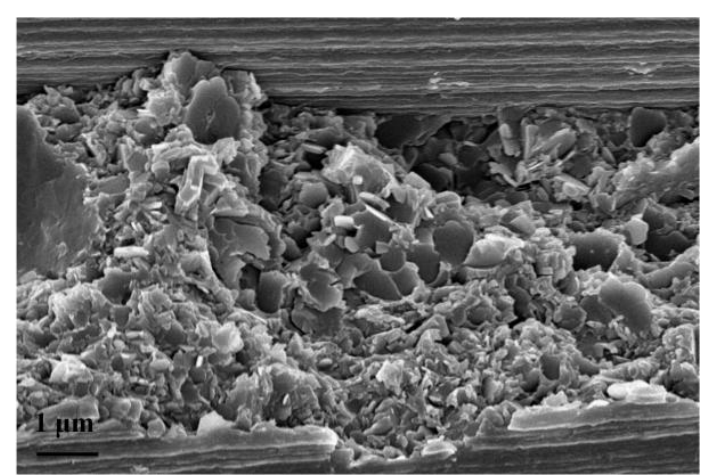

(b)

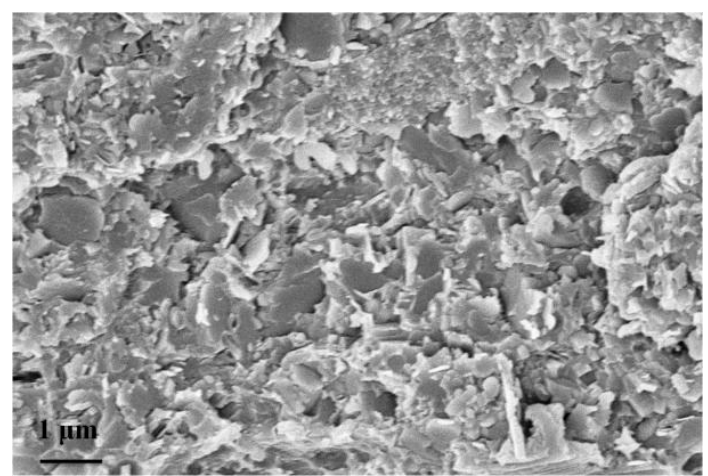

(d)

Figure 3 (a) and (b) are SEM images for MPCF/BN-20\%/epoxy and (b) is an enlarged image. (c) and (d) are SEM images for MPCF/mBN-20\%/epoxy and (d) is an enlarged 
one.

The thermal properties of MPCF/BN/epoxy and MPCF/mBN/epoxy composites with different volume fractions are measured by a Nanoflash method and the results including $\alpha$ and TC are presented in the Figure 4 (a) and (b). For the reference, the TC of MPCF/epoxy without BN fillers is $4.9 \mathrm{~W} /(\mathrm{mK})$. The calculated enhancement of TC (TCE\%) for MPCF/mBN/epoxy composites compared with the MPCF/BN/epoxy composites is follows the relations (1) and the results are listed in the Table 1.

$\mathrm{TCE} \%=\frac{\left(\mathrm{TC}_{\mathrm{MPCF} / \mathrm{mBN} / \mathrm{epoxy}}-\mathrm{TC}_{\mathrm{MPCF} / \mathrm{BN} / \text { epoxy }}\right)}{\mathrm{TC}_{\mathrm{MPCF} / \mathrm{BN} / \mathrm{epoxy}}} * 100 \%$

Table $1 \mathrm{TCE} \%$ for MPCF/mBN/epoxy composites compared with MPCF/BN/epoxy composites with different volume fraction of fillers.

\begin{tabular}{ccccc}
\hline Volume fractions of $\mathrm{mBN}$ & $5 \%(\mathrm{vol})$ & $10 \%(\mathrm{vol})$ & $15 \%(\mathrm{vol})$ & $20 \%(\mathrm{vol})$ \\
\hline TCE\% & $2.7 \%$ & $22.9 \%$ & $56.6 \%$ & $78.9 \%$ \\
\hline
\end{tabular}

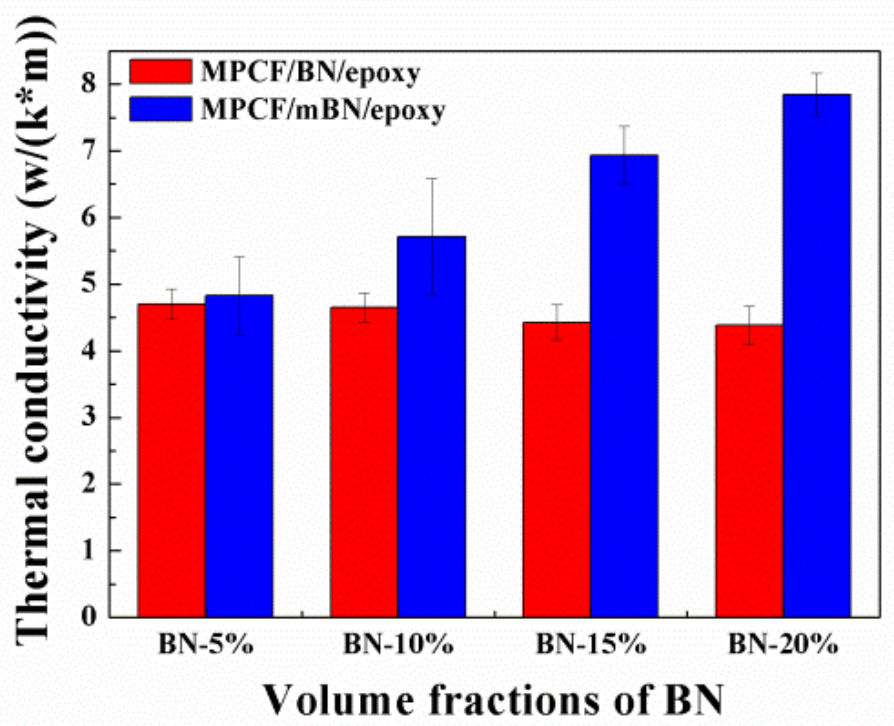

Figure 4. TC of MPCF/BN/epoxy and MPCF/mBN/epoxy composites with different volume fractions of fillers.

Results in Fig.4 illustrate that simply mixing BN with epoxy is not an effective 
way for MPCF epoxy matrix composites since the thermal properties of MPCF/BN/epoxy do not show obvious improvement as $f_{\mathrm{BN}}$ 's increasing. Instead, only after the surface treatment by KH550 for BN particles, the thermal properties of $\mathrm{MPCF} / \mathrm{mBN} / \mathrm{epoxy}$ increase obviously as the $f_{\mathrm{m}-\mathrm{BN}}$ increasing, especially the TC for MPCF/mBN-20\%/epoxy $\left(7.9 \mathrm{w} /\left(\mathrm{m}^{*} \mathrm{~K}\right)\right)$ whose TCE\% is $79 \%$ compared with MPCF/BN-20\%/epoxy $(4.4 \mathrm{w} /(\mathrm{m} * \mathrm{~K}))$ as presented in Table 1 . In order to explain the function of the surface treatment by KH550, it needs to retrospect the phonon's heat transfer mechanism in epoxy matrix. As inorganic fillers, the dispersion of $\mathrm{BN}$ particles in epoxy matrix creates huge amounts of interfaces as shown in the SEM images. The adhesion of BN particles and epoxy in the interfaces greatly determines interfacial thermal resistances which control phonon scattering and affect TC of the composite. According to the thermal property theory, the interfacial thermal resistance is generally classified in two ways: (1) by thermal contact resistance and (2) by thermal boundary resistance. Firstly, the thermal contact resistance usually results from several mismatches in the surface conditions of BN, epoxy and MPCF layers. Meanwhile, it also depends on how well these three materials are bonded together and the contact areas among three. Then the other part, the thermal boundary resistance, is caused by the mismatch in the vibration harmonics of phonons and/or the flow of phonons among BN, epoxy and MPCF. In the MPCF/BN/epoxy composites, with the volume fraction of BN increasing, these mismatches become gradually hard to adjust and simultaneously voids caused by the mechanical incompatibilities between BN and epoxy increase. ${ }^{[26]}$ As the results of these factors, the TC's enhancement for $\mathrm{MPCF} / \mathrm{BN} /$ epoxy is limited despite increasing BN's incorporation. In contrast, the results show that $\mathrm{mBN}$ treated by KH550 can reduce these mismatches and consequently modify the interface contacting. This modification can be explained by the formation of silanol groups $(\mathrm{Si}-\mathrm{OH})$ attached with the surface hydroxyls of $\mathrm{BN}$. With the formation of chemical bonding with epoxy matrix, the adhesion among epoxy, mBN and MPCF can be ameliorated and effectively decrease the possibility of interfacial scattering of phonons. Besides, with the small molecules of KH550 on the surfaces of $\mathrm{BN}$ particles, some voids perhaps can be filled and improve the 
compatibility between matrix and fillers which makes the packing more efficient especially for the case of $20 \%$ volume fractions. Therefore, the surface treatment by KH550 is an effective method for enhancing the TC of MPCF/mBN/epoxy composites.

In order to further study the function of KH550 from the perspective of epoxy molecular motions, the temperature dependence of TC for MPCF/BN-20\%/epoxy and $\mathrm{MPCF} / \mathrm{mBN}-20 \% /$ epoxy composites are investigated from 30 to $120{ }^{\circ} \mathrm{C}$. Before discussing the behavior of TC's temperature dependence, the study of the glass transition of epoxy matrix for these two composites is conducted by DMA to detect the interaction between fillers and matrix which helps to understand the behavior of TC's temperature dependence more straightforward.

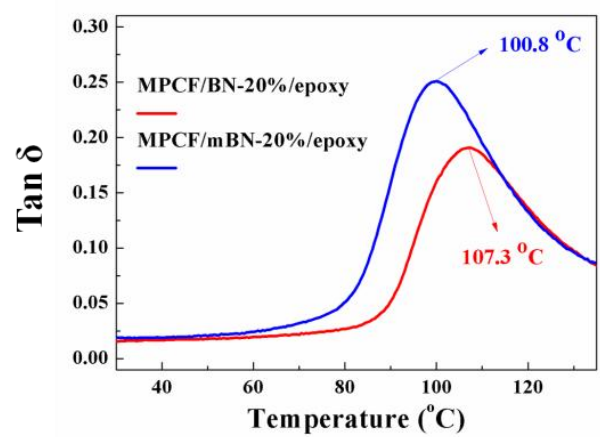

(a)

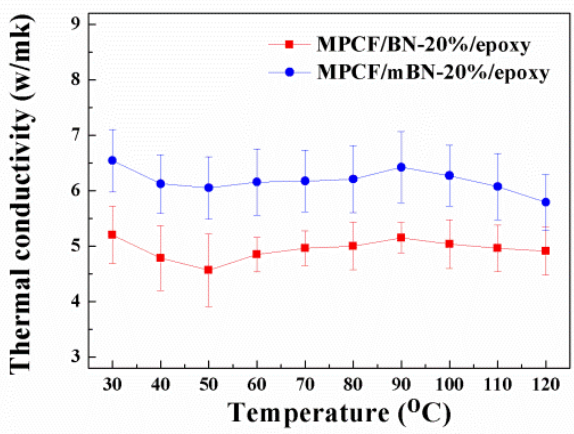

(b)

Figure 5. (a) Dynamic mechanical analysis for MPCF/BN-20\%/epoxy and MPCF/mBN-20\%/epoxy composites. (b) Temperature dependence of TC for the two composites.

The temperature of $\tan \delta$ peak for the composite is generally viewed as the glass transition for its polymer matrix in the DMA measurement. As illustrated in Fig.5 (a), $\mathrm{Tg}$ for MPCF/BN-20\%/epoxy are $107.3{ }^{\circ} \mathrm{C}$ which is about $7{ }^{\circ} \mathrm{C}$ higher than that of MPCF/mBN-20\%/epoxy $\left(100.8{ }^{\circ} \mathrm{C}\right)$ due to the addition of KH550. The shift of $\mathrm{Tg}$ in a composite is usually governed by two primate factors: the dispersion state of the fillers and the modified mobility of polymer in the interfacial region which is also known as the interaction between matrix and fillers. After KH550's treating, the 
organic-silance functions as the plastic which increases more free volume around the epoxy and consequently reduces the energy of the mobility for polymer segments. Besides, due to the base chemical property, KH550 may also influence the cross-linking degree of the epoxy which may also reduce Tg. Thus, the glass transition for MPCF/mBN-20\%/epoxy happens in advance at lower temperature than that of MPCF/BN-20\%/epoxy.

The temperature dependence of TC for two composites is presented in Fig.5 (b) and the effect of temperatures on TC can be divided into three stages: firstly TC for the two composites decreases when the temperature increases from 30 to $50{ }^{\circ} \mathrm{C}$ but later increases a bit as the temperature reaches $90{ }^{\circ} \mathrm{C}$. Differently, the change of TC for MPCF/mBN-20\%/epoxy is less obvious than that of MPCF/BN-20\%/epoxy as the temperature increasing. As shown by the equation (1), the obtained TC is dominated by three factors: thermal diffusivity, heat capacity and density. Thermal diffusivity and heat capacity are related with the phonons scattering and epoxy's segment motions. When the temperature increases from 30 to $50{ }^{\circ} \mathrm{C}$, phonon's scattering increases because of the thermally activated process which causes the thermal diffusivity decreases clearly, but in this procedure, the heat capacity does not increase clearly since epoxy's segment's motion is slight in such a temperature range which can also be found from DMA in the Fig. 5 (a). Thus, the reducing of thermal diffusivity directly leads to TC's decrease. But as the temperature increasing, in the second stage from 50 to $100{ }^{\circ} \mathrm{C}$, the heat capacity of composites increases which can not only offset the decrease of thermal diffusivity but also increase the global TC. TC's temperature dependence for two composites can reflect the cooperative molecular motions involving both intra- and intermolecular interactions and we use the activation energy as a tool to understand this procedure. ${ }^{[27]}$ The variation of activation energies for two composites can be obtained by fitting TC at different temperatures (50 to $100{ }^{\circ} \mathrm{C}$ ) with the well-known Arrhenius relation expressed in equation (3) and the fitting results are presented in the Fig.6.

$$
\mathrm{TC} \propto \frac{1}{\mathrm{~T}} \exp \left[-\frac{\mathrm{E}_{\mathrm{a}}}{\mathrm{k}_{\mathrm{b}} \mathrm{T}}\right]
$$


Where $E_{a}$ is activation energy, $T$ is the absolute temperature and $K_{b}$ is the Boltzmann constant. These parameters are significant to differentiate the nature of various conduction mechanisms. When the values of TC are applied from 50 to $100{ }^{\circ} \mathrm{C}, \mathrm{E}_{\mathrm{a}}$ for MPCF/BN/epoxy and MPCF/mBN/epoxy are obtained as 0.0527 and $0.0458 \mathrm{eV}$ as shown in the inset column graph of Fig.6. A smaller $E_{a}$ of MPCF/mBN/epoxy indicates that silanol groups $(\mathrm{Si}-\mathrm{OH})$ of $\mathrm{KH} 550$ in the interface region of $\mathrm{mBN}$ and epoxy enter into the epoxy cross-linking network which not only helps to form better adhesion but also fill some voids in the composite. This better adhesion and denser packing enable to decrease phonon scattering and stabilize the TC of the composite so that weaken the temperature effect compared with pristine BN's reinforcement.

As the temperature reaches over $100{ }^{\circ} \mathrm{C}$, the values of TC for MPCF/BN/epoxy and $\mathrm{MPCF} / \mathrm{mBN} / \mathrm{epoxy}$ decrease as the temperature increasing since composites start the glass transition. In this stage, the TC of MPCF/mBN/epoxy reduces more than that of MPCF/BN/epoxy for the increase of free volume around epoxy's segments after modified by KH550 as we have mentioned in DMA part. More free volumes make the epoxy segments easier to move which may increase thermal motion and consequently decreases TC for the composites. Thus in the third stage from 100 to $120{ }^{\circ} \mathrm{C}$, TC for two composites both decrease a bit and the value for MPCF/mBN/epoxy decreases more. But generally, two composites' TC do not reduce a lot and both of them show a good stability on TC during the whole temperature changing procedure due to the excellent thermal property of MPCF. 


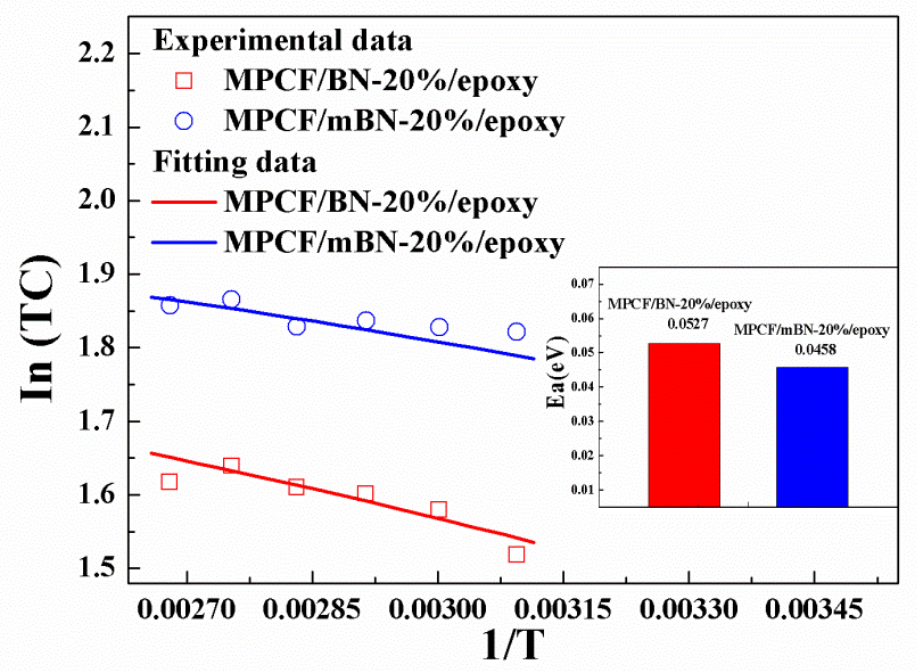

Figure $6 \ln$ (TC) verse temperatures for MPCF/BN/epoxy and MPCF/mBN/epoxy composites with $20 \%$ volume fraction fillers' incorporation. The inset graph shows the fitted active energies for two composites.

\section{Conclusion}

The MPCF/BN/epoxy composite achieves a high TC with a low volume fraction of BN. Moreover, after an effective surface treatment by KH550 coupling agent, MPCF/mBN-20\%/epoxy's TC has improved further and reaches $7.85 \mathrm{w} /(\mathrm{m} * \mathrm{~K})$ which has $79 \%$ increase compared with MPCF/BN/epoxy without surface treatment. Such a high enhancement on TC is attributed to the reinforced adhesion among $\mathrm{mBN}$, epoxy and MPCF achieved due to the silanol groups in KH550 which enables to reduce the thermal resistance as well as increase efficient packing between fillers and polymer matrix. Additionally, MPCF/BN-20\%/epoxy and MPCF/mBN-20\%/epoxy display a good stability on TC as the measurement temperature increasing because of the excellent thermal property of MPCF, before glass transition $\left(<100{ }^{\circ} \mathrm{C}\right)$, TC of $\mathrm{MPCF} / \mathrm{mBN}-20 \% / \mathrm{epoxy}$ is more stable to temperature change due to the KH550's surface treatment.

\section{Acknowledgement}


These works are carried out in the frame of the MUJU-Multimaterial mUltiphysics JUnctions-project, cofunded by the French National Research Agency "ANR" (project ANR-11-RMNP-0003).

\section{Reference}

[1] N. Burger, A. Laachachi, M. Ferriol, M. Lutz, V. Toniazzo, D. Ruch, Review of thermal conductivity in composites: Mechanisms, parameters and theory. Progress in polymer science. 2016.61. 1-28

[2] Hongyu Chen, Valeriy V. Ginzburg, Jian Yang, Yunfeng Yang, Wei Liu, Yan Huang, Libo Du, Bin Chen. Thermal conductivity of polymer-based composites: Fundamentals and application. Progress in Polymer Science. 2016. 59. 41-85

[3] Kojiro Uetani, Seisuke Ata, Shigeki Tomonoh, Takeo Yamada, Motoo Yumura, and Kenji Hata. Elastomeric Thermal Interface Materials with High Out-of-plane Thermal Conductivity from Carbon Fiber Fillers Vertically Aligned by Electrostatic Flocking. Advanced Materials 2014. 26, 5857-5862

[4] Xiaosong Huang, Fabrication and Properties of Carbon Fibers. Materials 2009, 2, 2369-2403

[5] Marilyn MInus, Satish Kumar. The processing, properties, and structure of carbon fibers. The Journal of the minerals, metals \& materials society (TMS). 2005. 57. 52-58

[6] Zhiqian Zhang, Yuwen Liu, Yudong Huang, Li Liu, Jianwen Bao. The effect of carbon-fiber surface properties on the electron-beam curing of epoxy-resin composites. Composites Science and Technology. 2002. 62. 331-337

[7] Ye Ji Noh, Seong Yun Kim. Synergistic improvement of thermal conductivity in polymer composites filled with pitch based carbon fiber and graphene nanoplatelets. Polymer Testing. 2015. 45. 132-138

[8] Kojiro Uetani, Seisuke Ata, Shigeki Tomonoh, Takeo Yamada, Motoo Yumura, and Kenji Hata. Elastomeric Thermal Interface Materials with High Out-of-plane Thermal Conductivity from Carbon Fiber Fillers Vertically Aligned by Electrostatic Flocking. Advanced Materials 2014. 26, 5857-5862

[9] A Shiota, CK Ober, Synthesis and curing of novel LC twin epoxy monomers for liquid crystal thermosets. Journal of Polymer Science Part A. 1996. 34. 1291-303 
[10] Shi-hui Song, Hideyuki Katagi, Yoshitaka Takezawa. Study on high thermal conductivity of mesogenic epoxy resin with spherulite structure. Polymer. 2012. 53. 4489-4492

[11] Zhidong Han, Alberto Fina. Thermal conductivity of carbon nanotubes and their polymer nanocomposites: A review. Progress in Polymer Science. 2011. 36. 914-944

[12] M. J. Biercuk, M. C. Llaguno, M. Radosavljevic, J. K. Hyun, A. T. Johnson and J. E. Fischer. Carbon nanotube composites for thermal management. Applied Physics Letters 2002. 80. 2767

[13] Aiping Yu, Palanisamy Ramesh, Xiaobo Sun, Elena Bekyarova, Mikhail E. Itkis, Robert C Haddon. Enhanced thermal conductivity in a hybrid graphite nanoplatelet-carbon nanotube filler for epoxy composites. Advanced Materials. 2008. 20. 4740-4744

[14] Aiping Yu, Palanisamy Ramesh, Mikhail E. Itkis, Elena Bekyarova, and Robert C. Haddon. Graphite Nanoplatelet-Epoxy Composite Thermal Interface Materials. The journal of physical chemistry C. 2007, 111, 7565-7569

[15] Martin Donnay, Spiros Tzavalas, Emmanuel Logakis. Boron nitride filled epoxy with improved thermal conductivity and dielectric breakdown strength. Composites Science and Technology. 2015, 110, 152-158

[16] Ziyin Lin, Andrew Mcnamara, Yan Liu, Kyoung-sik Moon, Ching-Ping Wong. Exfoliated hexagonal boron nitride-based polymer nanocomposite with enhanced thermal conductivity for electronic encapsulation. Composites Science and Technology. 2015, 90, 123-128

[17] Seran Choi, Jooheon Kim. Thermal conductivity of epoxy composites with a binary-particle system of aluminum oxide and aluminum nitride fillers. Composites Part B: Engineering. 2013, 51, 140-147

[18] Xingyi Huang, Tomonori lizuka, Pingkai Jiang, Yoshimichi Ohki and Toshikatsu Tanaka. Role of Interface on the Thermal Conductivity of Highly Filled Dielectric Epoxy/AIN Composites. The Journal of Physical Chemistry C, 2012, 116, 13629-13639

[19] Kamyar Pashayi, Hafez Raeisi Fard, Fengyuan Lai, Sushumna Iruvanti, Joel Plawsky and Theodorian Borca-Tasciuc. Self-constructed tree-shape high thermal conductivity nanosilver networks in epoxy. Nanoscale, 2014, 6, 4292-4296

[20] Seunggun Yu, Bo-In Park, Cheolmin Park, Soon Man Hong, Tae Hee Han and Chong Min Koo. RTA-Treated Carbon Fiber/Copper Core/Shell Hybrid for Thermally Conductive Composites. ACS Applied Materials and Interfaces 2014, 6, 7498-7503

[21] Xingyi Huang, Chunyi Zhi, Pingkai Jiang, Dmitri Golberg, Yoshio Bando, and Toshikatsu Tanaka. Polyhedral Oligosilsesquioxane-Modifi ed Boron 
Nitride Nanotube Based Epoxy Nanocomposites: An Ideal Dielectric Material with High Thermal Conductivity. Advanced Functional Materials. 2013, 23, 1824-1831

[22] Xingyi Huang, Tomonori Iizuka, Pingkai Jiang, Yoshimichi Ohki and Toshikatsu Tanaka. Role of Interface on the Thermal Conductivity of Highly Filled Dielectric Epoxy/AlN Composites. The Journal of Physical Chemistry C, 2012, 116 (25), 13629-13639

[23] Jinhong Yu, Xingyi Huang, Chao Wu, Xinfeng Wu, Genlin Wang, Pingkai Jiang. Interfacial modification of boron nitride nanoplatelets for epoxy composites with improved thermal properties. Polymer. 2012, 53, 471-480

[24] Wenying Zhou. Effect of coupling agents on the thermal conductivity of aluminum particle/epoxy resin composites. Journal of Materials Science. 2011, 46, 3883-3889

[25] Sabyasachi Gangulia, *, Ajit K. Roya, David P. Anderson, Improved thermal conductivity for chemically functionalized exfoliated graphite/epoxy composites. Carbon. 2008, 46, 806-817

[26] Stephen Ducharme. An inside-out approach to storing electrostatic energy. ACS Nano. 2009. 3. 2447-2450

[27] El Hasnaoui M, Graca MPF, Achour ME, Costa LC, Outzourhit A, Oueriagli A, et al. Effect of temperature on the electrical properties of copolymer/carbon black mixtures. Journal of Non-Crystalline Solids. 2010, 356, 1536-41 